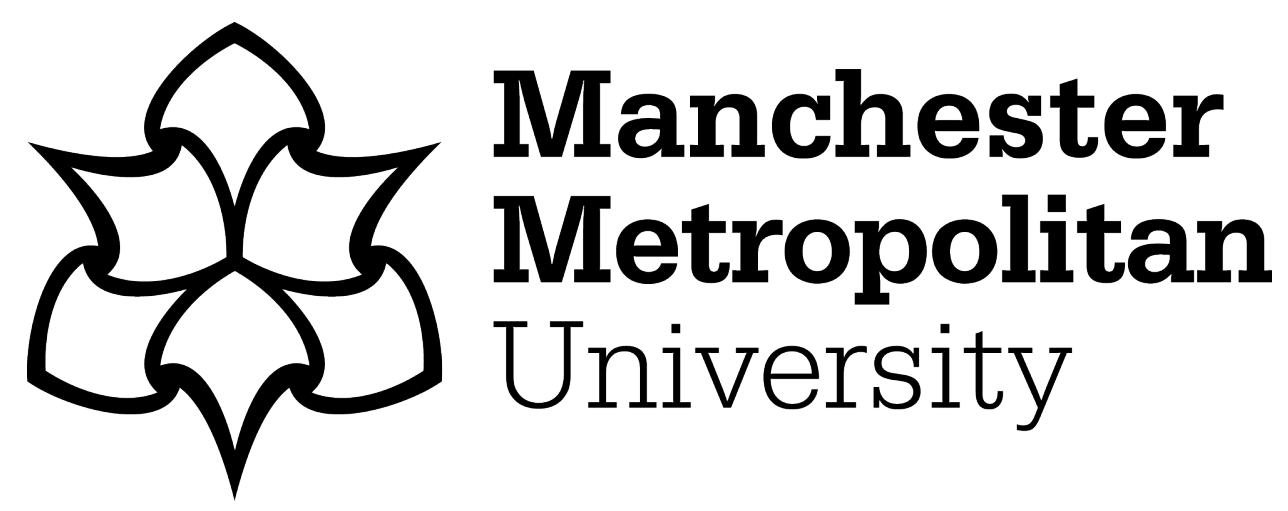

Seyedolhosseini, Atefesadat, Masoumi, Nasser, Modarressi, Mehdi and Karimian, Noushin (2019) Zone Based Control Methodology of Smart Indoor Lighting Systems Using Feedforward Neural Networks. In: 2018 9th International Symposium on Telecommunications (IST), 17 December 2018 - 19 December 2018, Tehran, Iran.

Downloaded from: https://e-space.mmu.ac.uk/626759/

Publisher: IEEE

DOI: https://doi.org/10.1109/istel.2018.8661118

Please cite the published version 


\section{Zone Based Control Methodology of Smart Indoor Lighting Systems Using Feedforward Neural Networks}

\author{
Atefesadat Seyedolhosseini \\ CST-LAB, Department of Electronics \\ School of Electrical and Computer \\ Engineering, College of Eng. University of \\ Tehran \\ Tehran, Iran \\ aseidolhosseini@yahoo.com
}

\author{
Nasser Masoumi \\ CST-LAB, Department of Electronics \\ School of Electrical and Computer, \\ Engineering, College of Eng., University of \\ Tehran \\ Tehran, Iran \\ nmasoumi@ut.ac.ir
}

Noushin Karimian

School of Engineering Science,

College of Eng., University of Tehran

Tehran, Iran

NKarimian@ut.ac.ir

\author{
Mehdi Modarress \\ School of Electrical and Computer \\ Engineering, College of Eng., University of \\ Tehran, and School of Computer Science, \\ Institute for Research in Fundamental \\ Sciences \\ Tehran, Iran \\ modarressi@ut.ac.ir
}

\begin{abstract}
A smart, accurate, and energy efficient control strategy to adjust dimming level of luminaires in an indoor environment is proposed in this paper. The control block in lighting system is nonlinear and time variant, since multiple reflections of objects and daylight variation are related to daytime and they can directly affect the system. According to the complexity of equations which model the lighting system, a control system based on Neural Network (NN) and learning machine is developed. By considering each zone as an independent structure, occupancy in each zone is added. In addition, photodetectors are placed at the work zones and hence increasing the accuracy. The occupancy condition for other zones in the environment are considered as bias to the inputs of the system. Therefore, multiple reflections in the environment are considered in the design of the proposed control method. Accuracy and system performance is improved by separation of control block for each zone as an autonomous control unit, whereas complexity of the system is reduced. The proposed design is evaluated in test beds developed using DIALux and MATLAB. The mean error varies according to the effect of zones on each other. The method is suitable for indoor environment that zones does not have common luminaires. The mean error in the case study that is not proper for the method does not exceed $20 \%$. Although, the error seems to be high but compared to the methods that have ceiling mount sensors is accurate and power and power efficient. Besides, the case with zones that has separated luminaires the mean error is less than $5 \%$.
\end{abstract}

Keywords-Dimming Level, Luminaire, Photodetector, Occupancy Condition, Neural Network, State Machine, Control Loop, Learning, Light Intensity, Light Uniformity

\section{INTRODUCTION}

Artificial lighting system plays an undeniable role in the amount of consumed energy, resident comfort, health, and security. Therefore, several standards have been developed to improve lighting system performance and crucial parameters such as light intensity and uniformity in a work area is specified by a pre-determined standard $[1,2]$. The effect of lighting system on human life, does not only results in new lighting technologies ssuch as solid-state lighting but also smart control systems to improve accuracy and system efficiency [3-11].

According to the standards, lighting system is divided in two main categories, indoor and output, where specific parameters are important for each type. In this paper, the primary focus is on the control strategy to efficiently adjust the dimming level of luminaires based on parameters determined in the standards for an indoor environment. Smart indoor systems are mostly implemented based on wireless sensor networks (WSNs) and on-battery nodes. Life time of on-battery nodes can be considered as an important parameter. Thus, the control system should work in a way to decrease the number of data transmission [9]. Indoor environment like offices may consists of multiple work zones. In addition, the amount of light intensity at any point is presented by lux (lx) and light uniformity (described by a percentage or ratio), are two important factors for indoor lighting.

Control strategy directly affects lighting system efficiency for a certain types of luminaires. Daylight and its variation during daytime and occupancy activation of an area are considerations that directly affect system performance. Some efforts have been made in order to improve lighting system proficiency [3-9]. Some scholars have modeled lighting system and extracted photodetector's output by equations. In a system with multiple light sources and due to the multiple reflections in an environment, complexity of lighting equations become additionally high. Therefore, control methods are also used in such systems and cost function based on the present constraints are defined.

A cost function based on light intensity and consumed energy with uniformity as a constraint was developed [3], where the linear relation between the dimming levels of light sources and photodetector's output have been assume. However, due to the presence of different objects with different surfaces in an environment and the multiple light surface reflections, the lighting is said to be a non-linear and time variant function. Consequently, control loop gain should be extracted based on cost function and light transport matrix in order to improve the accuracy of control system; light 
transport matrix [12] and it should also be mentioned that light transport matrix cannot be extracted theoretically. A control unit plays crucial role in the system, since the accuracy of linear modeling is limited and the cost function is analyzed based on linear models [3]. Similarly, a new method is proposed to recognize adjacent luminaires in the network [6]. This method experimentally extracts the light transport matrix and therefore reduced the consumed. The accuracy of dimming system is determined by control loop and several dimming steps is needed to converge which is undesirable in an on battery WSNs. As mentioned earlier, the number of transmission data should be optimized which is not achieved in the design proposed in [6]. Besides, the number of photodetectors are limited to the number of luminaires which adversely affect the system accuracy.

Considering linear relation between light source dimming levels and photo detector outputs is not a proper assumption in real environment, since reflections owing to different object with various reflection ratio make system nonlinear and authors in [8] based on linear relation modeled the system and developed a control algorithm. Hence, there is a need to define a proper cost function with constraints based on standards to improve accuracy of the whole system. The control system in lighting problem is a multiple-input multiple-output (MIMO) system, as authors mentioned in [9] and accordingly the analysis of such system is complex. Therefore, the researchers take the advantages of learning methods to control the lighting conditions in indoor work space. Although the assumption considered in [9] is right but the input and output of the system is applied inversely that makes design unsuitable for the control system. In this paper getting the advantages of neural network, light intensity of an indoor environment is controlled with desired uniformity determined by user request or standard. In the system designed in this paper, desirable light intensity and dimming level of the light sources is considered as an input and output, respectively. However, the input and output to the system is wrongly considered in [9]. Besides, the work done in [9] zoning effect is not taken into the account, which directly affect the error of the system. In this paper zone issue also is taken into account to control the dimming level of sources, that affect complexity, error, and power consumption of the lighting system.

One another negative point about the reviewed researches is that zoning effect is not added to the mentioned designs, which directly affect power consumption. Besides, sensor placement in lighting system plays an undeniable role on system accuracy and performance. In most outstanding researches photodetectors are placed near the luminaires and it not a proper idea as mentioned in [1], since sensor should be placed directly at work zones.

In this paper, smart and accurate control block for an indoor lighting system with multiple work zones is developed. Considering the fact that lighting system is nonlinear and time variant, the learning methods can be employed in order to optimize signal transmission in WSN based systems and improve accuracy. The proposed method is general and can be applied to any kind of lighting system; and it can also be implemented in a common processor with lower cost. The concept of lighting system, neural network and proposed control system, and test bed is discussed in section II. The proposed control system is evaluated by the developed test bed and the result is presented in section III. Section IV contains the conclusion of the whole research.

\section{ANALYTICAl StUdy: ILLUMinAtion CONTROL OF DIMMABLE LightiNG SYSTEM}

To design a proper control system, initially the lighting concept should be studied. The complexity of analytical equations, makes neural network an appropriate choice to apply to the system for controlling the light intensity. The control block and wisely selected test bed is discussed in this section, sequentially.

In the lighting system the desired intensity at each photodetector's output and occupancy condition of each zone is considered as an input. According to the control method decisions, the luminaire's dimming level is the output of the lighting system.

\section{A. Lighting System}

In a space, the light sources are classified into three parts; daylight, luminaires, and multiple reflections due to object surfaces and in this section these three parameters are evaluated. The photodetector's output current is as (1):

$$
y_{I}=I\left(L I_{T}\right)+v_{I}
$$

Where, the parameters $y_{I}, I\left(L I_{T}\right)$, and $v_{I}$ are output current of photodetector, the equivalent current of light intensity at the sensor's point, and sensor's dark current, respectively. The output current of photodetector at fully dark place $(0 l x)$ is referred to as dark current and should be taken into account in equations. Therefore, the equivalent light measured by the sensor can be modeled as (2):

$$
y=L I_{T}+v
$$

The variables $y, L I_{T}$, and $v$ are the measured light intensity by sensor in $l x$, the current light intensity at the point where that sensor is located, and extra noise that sensor is reported, respectively. The total amount of light in the environment, as mentioned earlier is the sum of three parameters.

$$
L I_{T}=L I_{F}+L I_{\text {Daylight }}+M L E
$$

The total light intensity at the sensor's point $\left(L I_{T}\right)$, is sum of light intensity generated by the lighting fixtures $\left(L I_{F}\right)$, effect of day light $\left(L I_{\text {Daylight }}\right)$, and multiple reflection due to the presence of many objects in the environment $(M L E)$. It is also worth mentioning that the percentage of reflected light from each surface is related to the transparency of objects in the environment. The parameter, MLE is the main parameter that results in a non-linear system. However, it is hard to calculate the effect of objects and multiple reflections that they led in the real environment. Besides, the effect of objects from one room to another one, varies and further increases the complexity of equations. Thus, the complexity of control system is increased by considering non-linear and time variant system. Learning algorithms diminish the elaboration by learning the behavior of the system and in this paper, the control block benefits from the advantages that neural network offers. Therefore, the number of data transmission is reduced and consequently battery life time of sensors is increased by the proposed control system. Once 
the system, is trained with specific data mount and network size, it can properly function and there is no need for calibration in a static system. This issue can be considered as one of the advantages of proposed control system, where the system is considered as non-linear with no extra complexity in the process of light setting.

Therefore, an efficient control system is developed in this paper to manage the lighting of an indoor environment. In the proposed design, both light intensity and uniformity are the determinative parameters of indoor lighting [1]. The proposed methodology is presented by an appropriate state machine to implement in various types of environment at processing elements. Learning methods is previously used to control lighting conditions of indoor environments; however, separation of work zones and placement of sensors are two important considerations added to the design and results in improvement of system performance, lower power consumption and data transmission. In the analytical methods, due to the calculation limitations, the number of photodetectors should equal to the luminaires. Fortunately, there is no limitation in the developed design and by increasing number of photodetectors, system accuracy is increased.

\section{B. Proposed Control System}

Lighting system is nonlinear and time variant and as discussed earlier theoretical formulation is intricate; owing to the complexity of coefficient calculation, a control system to accurately adjust dimming levels is proposed for indoor environment.

Neural network is mostly used as learning methods in nonlinear systems with various parameters and it is a suitable solution to control dimming level of lamps.

The control block is implemented by a state machine and it has 5 main states, as shown in Fig. 1. In the first state, referred to as initialization, communications and system elements are checked. The coordinator distributes a command through the network and each node respond back to the coordinator an ID and node type. The node type in the network can be occupancy sensor, LED, photodetector, and local controller. The network information of the system is gathered in the central coordinator. After finalizing the initialization state the flag called Init done is activated and the control system changes its state to data gathering which is named by Data G. All LEDs are requested to dim one by one in the data gathering state. The central coordinator sends a dim command to the first LED; the LED is dimmed from 0 to fully on in some specific time intervals, where the duration of time intervals is related to the time constant of photodetector and communication speed. The measured light intensity at each photodetector is reported to the local coordinator. The control block changes its state to the learning phase, if all LEDs are dimmed by activating Ddone flag.

By the gathered data, training the neural network starts and the size of network is chosen at a minimum determined level. The amount of data which is used to learn is considered as the minimum predetermined value.

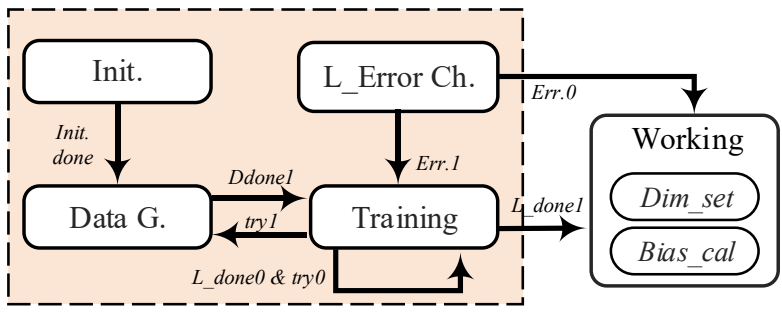

Fig. 1. Working states of developed smart lighting control system

If the network accuracy is not high enough, the size of the network will be increased. Each time the network size is increased, the accuracy is compared with that of the previous network size and if the error difference stays below 5\% and the accuracy is not at the desired region, the control system change its state to data gathering by activating the try flag. In this state the amount of data required to be fed to the neural network will initially be increased and if the accuracy is not improved, then the number of neurons is incremented in the following step. Increase in the number of neurons makes the neural network stronger; however, it results in higher computational power consumption. In the training phase, if the accuracy is enough, the flag named $L_{-}$done is activated and state machine goes to the working phase.

In the working phase every 30 minutes, photodetectors outputs are checked. If the outputs are varied from the predetermined value according to the luminaire's dimming levels, the variation is calculated and added to the desired intensity which is considered as an input to the system. Therefore, the luminaires are dimmed once more to improve the accuracy. Thus, the effect of occupancy and daylight are taken into account in the design, without increase of complexity.

Two different methods are investigated in this paper. In the first approach, the whole lighting system is considered as one neural network and therefore, the size of network should be considered high enough to achieve acceptable accuracy; in this method there is no local controller for each work zone and central controller manages all process and communication through the network. It's worth noting that, ranges of specific inputs (desired light intensity for different areas) may result in the same output which means that by different dimming levels, the same light intensity can be achieved in the areas under consideration. Therefore, the error is degraded by this issue, and in order to reduce the error in such networks, a design where each zone have specific network and state machine are proposed in this research. By applying the proposed method, occupancy as a separate parameter is also added to the control system and energy consumption hence, may decrease to a lower level, since there is no longer a need to dim all luminaires when occupancy is merely activated in one area. In the developed control block, specific light sources are selected to set the dimming level of each zone. Besides, the accuracy of the network is increased by the assumption that each zone has specific luminaires. It is illustrated in the next section that accuracy is increased using the above method. Also, network size is decreased since each area have only four luminaires and the network has three inputs (minimum, maximum and average light intensity at each work zone) and four outputs (dimming levels); however, in the method considering one network for the whole system, the network has 18 inputs and 12 outputs. 
TABLE I. Physical Parameters for The Considered Test Beds

\begin{tabular}{|c|c|c|}
\hline Parameter & Case \#1 & Case\#2 \\
\hline Room Area $\left(\mathbf{m}^{2}\right)$ & 20 & 225 \\
\hline Room Height & 2.74 & 2.7 \\
\hline Wall Reflection (\%) & 20 & 30 \\
\hline Window Size $\left(\mathbf{m}^{2}\right)$ & 2.42 & 2.96 \\
\hline Number of zones & 4 & 6 \\
\hline Number of Luminaires & 12 & 18 \\
\hline Work Zones Area $\left(\mathbf{m}^{2}\right)$ & $1.52 \times 0.75$ & $3 \times 1.5$ \\
\hline Work zones height (m) & 0.76 & 1 \\
\hline $\begin{array}{l}\text { Number of Sensors Per } \\
\text { Zone }\end{array}$ & 4 & 3 \\
\hline $\begin{array}{l}\text { Luminaire Part Number } \\
\text { in DIALux }\end{array}$ & $\begin{array}{l}\text { DIAL } 11 \\
\text { R2600/158 } \\
\text { P8 }\end{array}$ & $\begin{array}{l}\text { Zumtobel } 42182937 \\
\text { PERLUCE O LED2200- } \\
830 \text { D350 LDE IP50 WH } \\
\text { [STD] }\end{array}$ \\
\hline Sky Type & Overcast & Clear Sky \\
\hline
\end{tabular}

In static condition (without daylight variation), the proposed control system can function properly without sensors. This is because, after learning if environment parameters do not change there is no need to place sensors. Therefore, this design is suitable for on battery sensors in WSNs and static environments with limited life time. The new design contains six smaller neural networks with higher degree of accuracy.

\section{Test Beds}

The proposed control system is evaluated by two different test beds containing several dimmable luminaires in a DIALux. An indoor environment in both cases is considered as an office area, where the office has multiple working zones and daylight directly affecting the lighting condition. The daylight effect varies for each work area and based on requirement or time of a day, user may require different light intensity in the area.

Each room has different physical parameters, such as room dimensions, luminaires part number, etc. are reported in Table I. Besides, 3D view of each test bed is shown in Fig. 2. In the cases shown in Fig. 2 (a), each desk is considered as a single work zone that may have different occupancy condition and various visual task may be carried out there. Therefore, different intensity and uniformity may be required for each work zone in test beds.

As shown in Fig. 2, distance between work zones and luminaires' type vary between two test beds and thus the results may vary between these two cases as shown in next section.

Each room has a window that allow daylight to enter and therefore, each work zone receives different effect from the daylight. The effect of daylight is taken into account to simulate case studies just like real environment, daylight effect at each work zone in both case studies are reported in Error! Reference source not found.

Two different case studies are introduced in this part to evaluate the proposed method in this paper. Zone distance, number of zones, daylight effect and the luminaires are some of the important parameters that vary in test beds and located work zones in each test bed. Thus, the accuracy and efficiency of the proposed method for each case is compared.

\section{SiMULATION RESULTS}

The test setups described in section II, is used to evaluate the control system developed in this paper. At first the simple method considering one network for the whole system is evaluated and after that the output results regarding separate neural network for each zone is presented. The conventional and proposed method is implemented in MATLAB by the extracted lighting conditions from DIALux.

Considering one network for the whole system makes physical implementation more simple; the data transmission is increased and network size is also increased.

As mentioned in section II, according to the standards, the desired intensities at each sensor's locations are the input to the system and the luminaire's dimming level are the outputs. In Case \#1, shown in Fig. 2, 14 inputs and 12 outputs should be considered for the condition in controlling the whole system with one network and 18 inputs and 18 outputs for the case $\# 2$. Thus, the complexity and network size is incremented by increase in number of work zones and located sensors for the same accuracy needed.

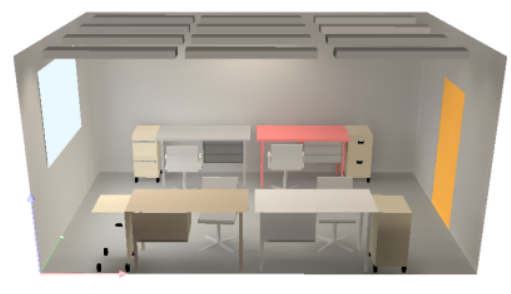

(a)

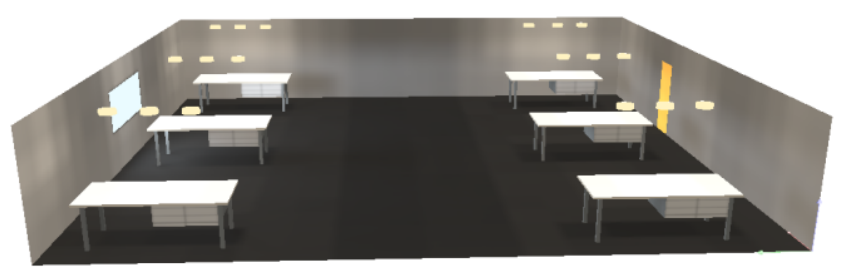

(b)

Fig. 2. 3D view of two different test beds considered to evaluate proposed method, (a) case \#1 and (b) case \#2

TABLE II. The MaXimum, Minimum, AND AVERAge MEASURED Light INTENSITY OF VARIOUS ZONES IN TEST BEDS WHEN ALL LUMINAIRES ARE OFF

\begin{tabular}{|c|c|c|c|c|c|c|c|}
\hline $\begin{array}{l}\text { Test } \\
\text { Bed } \\
\end{array}$ & Value & Z1 & $\mathbf{Z 2}$ & $\mathbf{Z 3}$ & Z4 & Z5 & Z6 \\
\hline \multirow{3}{*}{$\begin{array}{l}\text { ? } \\
\text { 0 } \\
0 \\
\text { \# }\end{array}$} & $\begin{array}{l}\text { Maximum } \\
\text { (lx) }\end{array}$ & 196 & 91 & 94 & 220 & - & - \\
\hline & $\begin{array}{l}\text { Minimum } \\
(\text { lx })\end{array}$ & 131 & 75 & 80 & 139 & - & - \\
\hline & $\begin{array}{c}\text { Average } \\
(I x)\end{array}$ & 284 & 115 & 107 & 357 & - & - \\
\hline \multirow{3}{*}{ 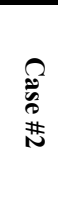 } & $\begin{array}{c}\text { Maximum } \\
(l x)\end{array}$ & 11 & 5.72 & 203 & 6.34 & 11 & 4.73 \\
\hline & $\begin{array}{c}\text { Minimum } \\
(l x)\end{array}$ & 7.91 & 5.52 & 4.3 & 5.61 & 7.59 & 3.72 \\
\hline & $\begin{array}{c}\text { Average } \\
(l x)\end{array}$ & 9.45 & 5.14 & 106.77 & 5.88 & 9.5 & 4.38 \\
\hline
\end{tabular}

A. Case \#1

In this part both approach (conventional and proposed method in this paper) for cases \#1 in Fig. 2 are evaluated and 
compared in different aspects. Considering one single network for the whole system, the error histogram for the entire network is shown in Fig. 3; the network is configured with 11 neurons. 16 desired intensities are considered as inputs and 12 luminaire's dimming level are delivered by the network as outputs.

Considering independent zones and local controllers for each zone the network error histogram for zone 1 with 4 inputs (desired intensities at sensor's locations) and 4 outputs (luminaire's dimming level) is shown in Fig. 4. As it is shown in Fig. 3 and Fig. 4, the accuracy is degraded by the zone separation process.

The network size is decreased by considering local controllers for each zone. Increase in network size requires higher computation efforts which increase the system cost.

In case $\# 1$, occupancy conditions of adjacent zones significantly affect lighting conditions, since they have common luminaires and distance between zones are low. When all zones occupancy condition is activated, the error is increased by $20 \%$, even by adding bias to the system, since the zones have common luminaires. However, compared to the previous efforts where the photodetectors where located adjacent to the luminaires at the ceiling, the accuracy is higher, as provided in Table III. There is more than $70 \%$ difference between the sensor's output in ceiling mounted photodetectors and work zone located sensors. Thus, the proposed method appears to be efficient compared to the previous methods.

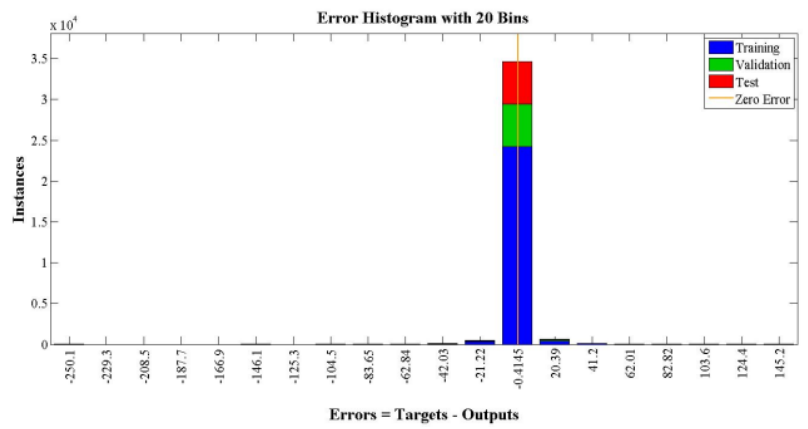

Fig. 3. Error histogram of feedforward neural network of Case \#1 with 11 neurons when considering one neural network for the whole system

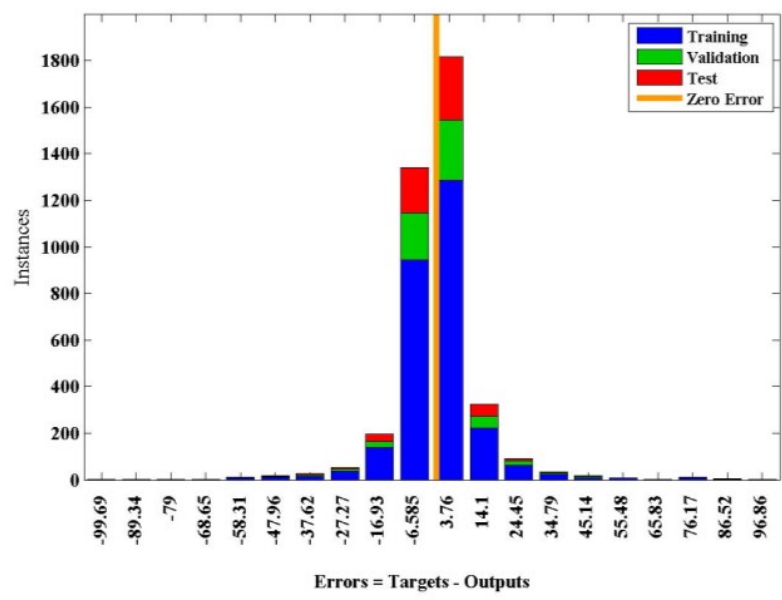

Fig. 4. Error histogram of zone 1 of case \#1 for the proposed method when the network size is equal to 8
TABLE III. DIFFERENCE BETWEEN MEASURED VALUE OF PHOTODETECTORS AT DIFFERENT LOCATIONS WHEN LUMINAIRES 4 AND 7 ARE FULLY DIMMED

\begin{tabular}{|l|l|}
\hline Location & Measured Intensity \\
\hline \multirow{4}{*}{ Zone \#1 } & 968.8 \\
\cline { 2 - 2 } & 839.6 \\
\cline { 2 - 2 } & 1054.9 \\
\cline { 2 - 2 } & 828.8 \\
\cline { 2 - 2 } & 947.2 \\
\hline \multirow{3}{*}{ Ceiling } & 204.5 \\
\cline { 2 - 2 } & 172.2 \\
\hline
\end{tabular}

\section{B. Case \#2}

For the systems with independent work zones, the proposed method works efficiently. The results for the system shown in Fig. 2 (b), is reported in this section.

Network's mean error for the conventional method by considering 11 neurons is $0.38 \%$; when considering one single network for the whole system. The network configuration error histogram is shown in Fig. 5.

The proposed method using local controller for each zone is useful as shown in Fig. 6. The mean error of the configured network has increased to $0.82 \%$, when all zones occupancy is activated the amount of intensity in the environment is considered as a bias to the system.

In the first case study, zones have common luminaires and they are placed adjacent to each other. In the next case study, zones have separate luminaires and located far from each other. In both case studies the method is compared to the conventional methods that proposed in previous works.

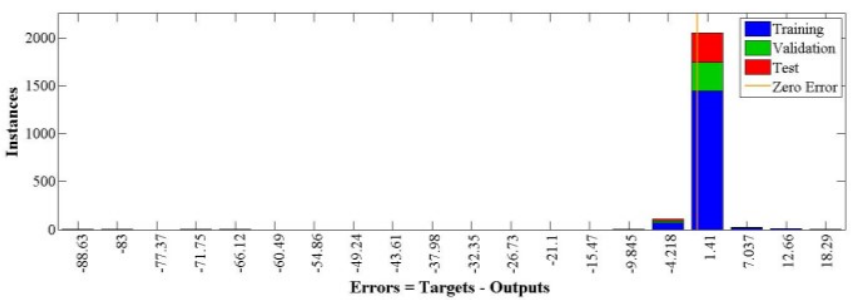

Fig. 5. The error histogram of the outputs for case \#2 when the whole system is configured with one network for the feedforward network with 11 neurons

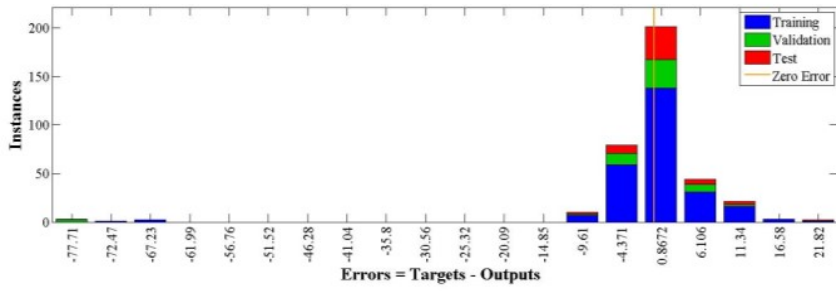

Fig. 6. The error histogram of configured network for the proposed method for zone \#1 in case \#2 for the feedforward network with 5 neurons

\section{CONCLUSION}

In this paper, a smart lighting control system suitable for indoor environment with multiple number of luminaires is proposed. The proposed control strategy is smart and energy 
efficient, since nonlinear and time variant characteristics of a real lighting system is taken into account. A state machine is developed to advance the performance of controller. The controller performance is improved by taking the advantages of learning methods. Neural network is applied to add learning capability. The proposed controller which is based on neural network is suitable for WSNs with on-battery nodes and functions for vast number of work zones. The system learns about the environment and thus in static condition sensors can be removed. In the proposed design, one network is specified for each work zone and therefore, the network size is decreased. The complexity of the system is diminished by reducing network size for each zone and the accuracy is improved by smaller network size and lower number of data set in the learning phase of the proposed method. The amount of consumed energy is decreased, since lower number of light sources are involved to light each work zone. The number of neural outputs is decreased by the proposed method compared to the conventional methods, due to the lower number of luminaires. The number of inputs is reduced which in turn reduced the complexity of the system. The proposed method is suitable for cases where work zones have separate luminaires. The proposed method is evaluated in an indoor environment and the test beds are implemented in DIALUX software. The results are processed in MATLAB and reported. The mean error in the case study that is not proper for the method does not exceed $20 \%$. Although, the error seems to be high but compared to the methods that have ceiling mount sensors is accurate and power and power efficient. Besides, the case with zones that has separated luminaires the mean error is less than $5 \%$.

\section{REFERENCES}

[1] Light and Lighting - Lighting of Work Places - Part I: Indoor Work Places, European Standard, EN 12464-1, 2011.

[2] Lighting of Work Places - Part II: Outdoor Work Places, European Standards, EN 12464-2, 2014.

[3] S. Afshari, S. Mishra, A. Julius, F. Lizarralde, J. D. Wason, J. T. Wen, "Modeling and Control of Color Tunable Lighting Systems," Elsevier Journal on Energy and Building, no. 68, pp. 242-253, 2014.

[4] S. Borile, A. Pandharipande, D. Caicedo, A. Cenedese, and L. Schenato, "An Identification Approach to Lighting Control," European Control Conference (ECC), 2016.

[5] Z. Kaleem, T. M. Yoon, and Ch. Lee, "Energy Efficient Outdoor Light Monitoring and Control Architecture Usign Embedded Systems," IEEE Embedded Systems Letters, vol. 8, no. 1, Mar. 2016.

[6] S. Afshari and S. Mishra, "A Plug-and-Play Realization of Decentralized Feedback Control for Smart Lighting Systems," IEEE Trans. On Control Systems Technology, vol. 24, no. 4, Jul. 2016.

[7] S. Li, A. Pandharipande, and F. M. J. Willems, "Daylight Sensing LED Lighting System," IEEE Sensors Journal, vol. 16, no. 9, May 2016.

[8] D. Caicedo and A. Pandharipande, "Distributed Illumination Control With Local Sensing and Actuation in Networked Lighting Systems," IEEE Sensors Journal, vol. 13, no. 3, Mar. 2013.

[9] D. Tran and Y. Kh. Tan, "Sensorless Illumination Control of A Networked LED-Lighting System Using Feedforward Neural Network," IEEE Trans. On Industrial Electronics, vol. 61, no. 4, Apr. 2014.

[10] F. Viani, A. Polo, P. Garofalo, N. Anselmi, M. Salucci, and E. Giarola, "Evolutionary Optimization Applied to Wireless Smart Lighting in Energy-Efficient Museums," IEEE Sensors Journal, vol. 17 , no. 5, 2017.

[11] I. Chew, V. Kalavally, Ch. P. Tan, and J. Parkkinen, "A SpectrallyTunable Smart LED Lighting System with Closed-Loop Control," IEEE Sensnors Journal, vol. 16, no. 11, 2016.

[12] J. Foely, A. V. Dam, and S. K. Feiner. Computer Graphics: Principles and Practice in C. $2^{\text {nd }}$ Edition, Addison-Wesley Professional, 1995. 